IUHET 295

COLBY-95-01

\title{
ELLIPTICAL SQUEEZED STATES AND RYDBERG WAVE PACKETS
}

\author{
Robert Bluhm, ${ }^{a}$ V. Alan Kostelecký, ${ }^{b}$ and Bogdan Tudose ${ }^{b}$ \\ ${ }^{a}$ Physics Department \\ Colby College \\ Waterville, ME 04901, U.S.A. \\ ${ }^{b}$ Physics Department \\ Indiana University \\ Bloomington, IN 47405, U.S.A.
}

\begin{abstract}
We present a theoretical construction for closest-to-classical wave packets localized in both angular and radial coordinates and moving on a keplerian orbit. The method produces a family of elliptical squeezed states for the planar Coulomb problem that minimize appropriate uncertainty relations in radial and angular coordinates. The time evolution of these states is studied for orbits with different semimajor axes and eccentricities. The elliptical squeezed states may be useful for a description of the motion of Rydberg wave packets excited by shortpulsed lasers in the presence of external fields, which experiments are attempting to produce. We outline an extension of the method to include certain effects of quantum defects appearing in the alkali-metal atoms used in experiments.
\end{abstract}

To appear in the September 1995 issue of Physical Review A 


\section{INTRODUCTION}

Coherent states [1] are candidate quantum-mechanical states for probing the interface between classical and quantum mechanics. Obtaining appropriate coherent states for a given quantum situation can be a difficult task, even for apparently simple systems. For example, although Schrödinger succeeded in finding exact nonspreading coherent states for the harmonic oscillator [2], he was unable to find an analogous solution for the Coulomb potential [3]. This problem has been discussed by many authors, and indeed it is now known for the Coulomb case that there are no exact solutions representing localized nonspreading packets following the classical motion 凹, 5, 6, 6, 8, 9, 10].

Given the impossibility of solving the original Schrödinger problem, one can seek instead closest-to-classical wave packets for the Coulomb potential without imposing the condition that the expectation values follow the exact classical motion for all time. It is natural to consider first a simplified version of this problem in which attention is restricted to the radial part of the motion, with a fixed (say, p-state) angular wave function. Physically, a wave function of this type results when a short laser pulse incident on a Rydberg atom excites a coherent superposition of energy states with identical angular-momentum quantum numbers.

It turns out that a kind of squeezed state [11] called a radial squeezed state (RSS) provides a good description of a closest-to-classical radial packet of this type [12, [13]. Such states minimize the uncertainty relation in a set of variables for which the radial Coulomb problem takes a form similar to that of an oscillator. An oscillator squeezed state is like a coherent state in that it follows the classical motion for all time. However, it does not maintain its shape, instead having a position-space spread that oscillates periodically [14]. It can be shown that the corresponding Coulomb RSS has radial motion following the closest-to-classical motion along a keplerian ellipse. As is characteristic of a squeezed state, the RSS exhibits oscillations in the uncertainty product and ratio as a function of time.

Radial wave packets with p-state angular distributions have been experimentally produced using short-pulsed lasers to excite a coherent superposition of Rydberg 
states [15, 16, 17, 18, 19, 20]. The resulting localized Rydberg wave packets have features that match some of those of a classical particle moving in a Coulomb potential. For example, the initial motion is periodic with the classical keplerian period. While radial Rydberg wave packets initially follow the classical motion, they also exhibit effects due to quantum-mechanical interference. For instance, at times beyond a few classical periods they collapse and undergo a cycle of fractional/full revivals 21, 22, 23, 24]. Indeed, at still larger times, well beyond the appearance of the first full revival, the quantum-interference effects cause radial wave packets to undergo a new cycle of fractional/full superrevivals [25, 26]. Eventually, at times large compared with the superrevival time scale, the wave packets spontaneously decay or lose their coherence through other decay processes.

The RSS description reproduces the primary features in the motion of a Rydberg wave packet produced by a short-pulsed laser, including the revival and superrevival structures. Moreover, the RSS approach has a number of attractive features. A simple example is the shape of an RSS wave packet, which, unlike a simple gaussian, is asymmetrical along the radial direction. This asymmetry is desirable in a theoretical description of a packet produced with a transform-limited laser pulse exciting a superposition of unequally spaced Coulomb energy levels.

It is natural to ask whether this analytical description of the radial Coulomb problem can be extended to incorporate the angular dependence characteristic of a classical particle moving in a keplerian ellipse. The issue is of particular interest at present because experiments are currently attempting to produce Rydberg wave packets that move along elliptical trajectories.

The purpose of the present paper is to address this question. We provide here a planar extension of the RSS construction to a description of minimum-uncertainty wave packets moving along a keplerian ellipse, and we discuss some of the key features of the solution. Like the RSS, the packets derived here are squeezed states. We call them elliptical squeezed states (ESS).

Unlike purely radial packets, which have p-state angular distributions, experimental production of wave packets localized in angular coordinates necessarily involves generating a superposition of $l$ states. Superpositions of different $l$ states in a man- 
ifold of fixed $n$ have been produced [27]. Although a packet of this kind is indeed localized in angular variables, the degeneracy of the $l$ states in hydrogen means that it is stationary and hence fails to follow the classical motion.

The production of a wave packet localized in both radial and angular variables requires creating a superposition of both $n$ and $l$ states. To date, the only experiments that have detected periodic motion of a Rydberg wave packet with the classical keplerian period are those performed with radial packets, which have fixed $l$. However, a technique for producing a superposition of both $n$ and $l$ states has been suggested 28]. The proposal involves making use of a short electric field to convert an angular state into a localized Rydberg wave packet. Once produced, such a wave packet would move on a circular orbit. If an additional weak electric field is present, a wave packet moving on an ellipse of arbitrary eccentricity could be produced. Its motion would have features similar to those of the ESS we present here.

We begin our derivation of the ESS with a discussion of the planar RSS appropriate for the keplerian motion in Sec. II. The next task, performed in Sec. IIIA, is to obtain appropriate quantum-mechanical operators describing the angular position and angular momentum of a particle in a central potential. In the remainder of Sec. III, we proceed to the construction of a wave packet, called a circular squeezed state (CSS), that minimizes the uncertainty product for the angular variables, and we discuss some of its properties.

Section IV provides the construction of the ESS by minimizing the appropriate uncertainty relations. At the initial time, the ESS may be written as the products of RSS with CSS. Since the ESS naturally involve a superposition over both $l$ and $n$ quantum numbers, localization in both the angular and radial coordinates is achieved. In this section, we also obtain expectation values for relevant physical quantities and discuss the time evolution. The ESS move with classical keplerian periodicity along an elliptical orbit of fixed mean eccentricity and semimajor axis.

Since experiments are often performed in alkali-metal atoms rather than with hydrogen, it can be important to allow for modifications due to quantum defects. 
Some of these are treated in Sec. V. Finally, Sec. VI summarizes our results.

\section{PLANAR RADIAL SQUEEZED STATES}

In this section, we outline the construction of the radial squeezed states for motion in a plane. Although the results differ in detail, much of the analysis is similar to ref. [13], to which the reader is referred for a more complete treatment.

The RSS construction begins with the classical effective radial hamiltonian for the Coulomb potential written in terms of the radial variables $r$ and $p_{r}$ and converts it to an oscillator description in terms of new variables $R$ and $P$. The resulting classical problem is then quantized, and wave functions are obtained minimizing the quantum uncertainty relation obeyed by $R$ and $P$. These wave functions are the RSS. One motivation for this construction is that the minimum uncertainty of the RSS with respect to oscillator coordinates incorporates some of the attractive features of oscillator squeezed states, while maintaining the RSS time evolution controlled by the Schrödinger equation with a Coulomb potential.

Classically, the effective radial planar hamiltonian for a particle in a Coulomb potential is

$$
H \equiv \frac{1}{2} p_{r}^{2}+\frac{l^{2}}{2 r^{2}}-\frac{1}{r}=E,
$$

where $p_{r}$ is the radial momentum and $E$ is the energy. For $E<0$, the radial motion is oscillatory between the values $r_{1,2}$ given by $r_{1,2}=a(1 \pm e)$, where $a=1 / 2|E|$ is the semimajor axis of the orbital ellipse and $e=\left(1-2 l^{2}|E|\right)^{1 / 2}$ is the eccentricity, with $l$ the constant classical angular momentum. The classical orbital period is given by $T_{\mathrm{cl}}=\pi /(2|E|)^{3 / 2}$.

The conversion to an oscillator description is via the variable change [6]

$$
R=\frac{1}{r}-\frac{1}{l^{2}} \quad, \quad P=p_{r} .
$$

In terms of $R$ and $P$, the equation $H=E$ becomes

$$
\frac{1}{2} P^{2}+\frac{1}{2} l^{2} R^{2}=\frac{1}{2} e^{2},
$$

which has the form of an energy equation for an oscillator. 
Promoting $R$ and $P$ to the status of quantum-mechanical operators $R=\frac{1}{r}-\frac{1}{l^{2}}$ and $P \equiv p_{r}=-i\left(\partial_{r}+1 / 2 r\right)$, where $\left[r, p_{r}\right]=i$, we obtain the commutation relation $[R, P]=-i / r^{2}$. The RSS are the wave functions $\psi(r)$ minimizing the corresponding uncertainty relation

$$
\Delta R \Delta P \geq \frac{1}{2}\left\langle\frac{1}{r^{2}}\right\rangle
$$

at fixed time.

The minimum-uncertainty wave packets are given by

$$
\psi(r)=N_{1} r^{\alpha} \exp \left[-\gamma_{0} r-i \gamma_{1} r\right]
$$

where the normalization constant is $N_{1}=\left(2 \gamma_{0}\right)^{\alpha+1} /[\Gamma(2 \alpha+2)]^{1 / 2}$. The parameters $\alpha, \gamma_{0}$, and $\gamma_{1}$ are given in terms of the squeezing

$$
S=\frac{\Delta R}{\Delta P}=\frac{2(\Delta R)^{2}}{\left\langle\frac{1}{r^{2}}\right\rangle}
$$

and expectations of the operators $1 / r$ and $p_{r}$ by

$$
\alpha=\frac{1}{S}-\frac{1}{2} \quad, \quad \gamma_{0}=\frac{1}{S}\left\langle\frac{1}{r}\right\rangle \quad, \quad \gamma_{1}=-\left\langle p_{r}\right\rangle
$$

The wave functions $\psi(r)$ form a three-parameter family of planar RSS. The radial part of the classical motion is uniquely determined by two parameters specifying a point in the $r-p_{r}$ phase space. However, three parameters are needed to fix the quantum-mechanical solution $\psi(r)$ because, in addition to specifying $\langle r\rangle$ and $\left\langle p_{r}\right\rangle$, it is necessary to provide information about the spread of the packet. This can be done, for instance, by fixing the mean energy $\langle H\rangle$.

The RSS discussed in refs. [12, 13] can be used to describe a radial Rydberg wave packet with p-state angular distribution produced by a single short laser pulse. The corresponding three RSS parameters for this situation are determined by matching the RSS expectation values for $\langle H\rangle,\langle r\rangle$, and $\left\langle p_{r}\right\rangle$ to mean values for the Rydberg wave packet at the outer apsidal point. Three experimental quantities control this match. The frequency of the laser pulse determines the mean energy of the wave packet. The time delay after the excitation of the wave packet determines the location of the packet. Finally, the duration of the laser pulse fixes the spread of the wave packet. 
Our purpose in the present work is to obtain wave packets (the ESS) that follow the classical keplerian motion along an ellipse. This implies incorporating nontrivial angular dependence into the analysis, so quantities such as the mean energy depend on both radial and angular coordinates. Again, certain expectation values specify the ESS parameters. However, the coordinate dependence is sufficiently intertwined that it is impossible to treat independently the determination of the three planar RSS parameters $\alpha, \gamma_{0}$, and $\gamma_{1}$ of Eq. (5). For this reason, we defer until Sec. IV a discussion of this topic.

\section{CIRCULAR SQUEEZED STATES}

The goal of this section is to obtain the circular squeezed states, which can be viewed as squeezed states for angular coordinates in the plane. Sec. IIIA contains some remarks about the choice of angular coordinates. Sec. IIIB derives the CSS, while Sec. IIIC examines properties of the CSS.

\section{A. Quantum Angular Variables}

The effective angular hamiltonian for planar motion is the square $L^{2}$ of the angular momentum operator $L$. There are, however, subtleties involved in the choice of the corresponding angular coordinates to use in the quantum description. This subsection briefly discusses some of the issues directly relevant to the construction of the CSS and provides the definitions and conventions we have adopted. For more details and a guide to the early literature on this topic, we refer the reader to the review [29].

Among the possible choices for the angular coordinate are the continuous variable $\phi_{c}$ with $-\infty<\phi_{c}<\infty$ and the periodic variable $\phi_{p}$ with $-\pi \leq \phi_{p}<\pi$. Both these choices present problems at the quantum level. The continuous variable $\phi_{c}$ is not periodic, and hence is not an observable in the Hilbert subspace of functions for which $L=-i \partial_{\phi}$ is hermitian under a conventional inner product. In contrast, $\phi_{p}$ is periodic but is discontinous at $\phi_{p}=\pi$, which causes unusual effects with derivative

operators such as the angular momentum. For instance, direct substitution into the commutation relation $\left[\phi_{p}, L\right]$ generates $\delta$-function contributions. 
These difficulties can be avoided by choosing instead angular coordinates that are both periodic and continuous. However, a single such quantity is insufficient to specify uniquely a point on a circle, since periodicity implies the existence of extrema and hence no single quantity can be one-to-one. One possibility [30] is to use two angular coordinates, $\cos \phi$ and $\sin \phi$, where $\phi$ is either $\phi_{c}$ or $\phi_{p}$. Classically, this is a natural choice since it corresponds to the identification $(x, y) \rightarrow(\cos \phi, \sin \phi)$ on a unit circle.

The corresponding quantum operators can be defined through their matrix elements in the Hilbert space. We take as the inner product the definition

$$
\left\langle\psi_{1} \mid \psi_{2}\right\rangle=\int_{-\pi}^{\pi} d \phi \psi_{1}^{*} \psi_{2}
$$

Matrix elements of the quantum operators $\widehat{\cos } \phi$ and $\widehat{\sin } \phi$ are then given by insertion of the coordinate functions $\cos \phi$ and $\sin \phi$ in the inner product (8).

The angular operators obey the nonlinear relation $\widehat{\sin \phi} \phi^{2}+\widehat{\cos } \phi^{2}=I$, where $I$ is the identity operator. Note that using one of the two angular coordinates along with the sign of the other suffices to determine a unique location on a circle. However, this choice is not smooth. Taken together, the pair of operators $\widehat{\cos } \phi, \widehat{\sin } \phi$ have all the features needed for a quantum-mechanical treatment.

The quantum angular-coordinate operators $\widehat{\cos } \phi$ and $\widehat{\sin \phi}$ and the angular momentum $L$ are intertwined by the commutation relations

$$
[\widehat{\cos } \phi, L]=-i \widehat{\sin \phi} \quad, \quad[\widehat{\sin \phi}, L]=i \widehat{\cos \phi} \quad, \quad[\widehat{\cos \phi}, \widehat{\sin \phi}]=0
$$

The uncertainty relations following from these equations are

$$
\begin{aligned}
& \Delta \widehat{\cos \phi} \Delta L \geq \frac{1}{2}|\langle\widehat{\sin \phi}\rangle|, \\
& \Delta \widehat{\sin \phi} \Delta L \geq \frac{1}{2}|\langle\widehat{\cos \phi}\rangle|
\end{aligned}
$$

and

$$
\Delta \widehat{\sin \phi} \Delta \widehat{\cos \phi} \geq 0
$$

The last of these relations shows that $\widehat{\cos } \phi$ and $\widehat{\sin } \phi$ can be specified simultaneously to arbitrary precision, which is intuitively reasonable since both quantities are needed to determine the location of the quantum particle. 
For simplicity, in the remainder of this paper we adopt the usual convention of writing $\widehat{\cos } \phi$ as $\cos \phi$ and $\widehat{\sin } \phi$ as $\sin \phi$, leaving the context to determine whether an operator or a function is intended.

\section{B. Construction of CSS}

We begin by seeking a minimum-uncertainty state that is centered about $\phi=0$. Circular symmetry then implies the conditions

$$
\langle\sin \phi\rangle=0 \quad, \quad\langle\cos \phi\rangle>0 \quad .
$$

This means that the uncertainty relation (11) provides the only nontrivial constraint. A wave packet $\chi(\phi)$ minimizing this relation must obey the differential equation

$$
(\sin \phi) \chi=-i \frac{1}{\delta}(L-\langle L\rangle) \chi
$$

where the squeezing $\delta$ in the angular coordinates is a real constant given by

$$
\frac{1}{\delta} \equiv \frac{\Delta \sin \phi}{\Delta L}=\frac{2(\Delta \sin \phi)^{2}}{|\langle\cos \phi\rangle|} \geq 0 \text {. }
$$

The solution to Eq. (14) is

$$
\chi(\phi)=N_{2} \exp (\delta \cos \phi+i \beta \phi),
$$

where we have defined the real parameter $\beta=\langle L\rangle$. Note that $\beta$ must be integer for the $\chi(\phi)$ to be single valued. This is a special case of the general result that minimumuncertainty angular packets must have integer angular-momentum expectations [31. The normalization constant $N_{2}$ is given by

$$
N_{2}=\sqrt{\frac{1}{2 \pi I_{0}(2 \delta)}},
$$

where $I_{n}(z)$ is a modified Bessel function of the first kind. The two-parameter family of states (16) are the CSS. They have previously entered the literature in the context of uncertainty relations for phase and angle variables [29]. In addition to $\langle L\rangle=\beta$ and $\langle\sin \theta\rangle=0$, already imposed above, we find

$$
\langle\cos \phi\rangle=\frac{I_{1}(2 \delta)}{I_{0}(2 \delta)} \geq 0
$$


as expected.

By construction, the CSS (16) have magnitude peaking at $\phi=0$. It is shown in Sec. IV that this is appropriate for use in deriving packets moving along an ellipse aligned with outer apsidal point at $\phi=0$, which is the orientation most likely to be relevant in future experiments. However, modified CSS with magnitude peaking at some angle $\phi=\phi_{0}$ instead would be relevant for packets moving along an ellipse of arbitrary orientation. The remainder of this subsection concerns these modified CSS.

We first remark that imposing $\langle\cos \phi\rangle<0$ instead of Eq. (13) results in a sign change of the inverse squeezing $\delta$ and hence in a packet of the same shape as Eq. (16) but centered about $\phi=\pi$. Another possibility is to seek a configuration with $\langle\cos \phi\rangle=0$ instead of (13), whereupon the interesting constraint becomes Eq. (11) instead of (10). The solution is again of the form (16) but with $\sin \phi$ replaced by $\cos \phi$ in the exponential, representing a packet centered at $\phi= \pm \pi / 2$ according to the sign of $\langle\sin \phi\rangle$.

One approach to the construction of a packet centered at an arbitrary angle $\phi_{0}$ is to take advantage of rotational covariance. Implicit in the use of $\cos \phi$ and $\sin \phi$ as angular variables is a choice of origin for $\phi$, or, equivalently, a choice of orientation for the coordinate axes in the plane. However, the effective angular hamiltonian $L^{2}$ has $\mathrm{U}(1)$-rotational invariance corresponding to independence of the choice of origin. A rotation by an angle $\phi_{0}$ may be implemented by a translation in the continuous coordinate, $\phi_{c} \rightarrow \phi_{c}-\phi_{0}$. This leaves invariant all physical observables since the inner product (8) is invariant on functions of the periodic coordinates $\sin \theta$ and $\cos \theta$.

The conditions (13) and the solution (16) break this $U(1)$ symmetry. A U(1) rotation of (16) by $\phi \rightarrow \phi-\phi_{0}$ produces a different packet with maximum centered at $\phi_{0}$ :

$$
\chi\left(\phi, \phi_{0}\right)=N_{2} \exp \left[\delta \cos \left(\phi-\phi_{0}\right)+i \beta\left(\phi-\phi_{0}\right)\right],
$$

Instead of Eq. (10), this packet minimizes an uncertainty relation between a new angular-coordinate operator $\cos \left(\phi-\phi_{0}\right)$ and the angular-momentum operator $L$.

An interesting issue is whether there is a meaningful way to minimize both (10) and (11). Simultaneous extremization of both relations is impossible. Furthermore, 
various combinations could be chosen for minimization, producing a variety of interpolations between the solutions found at $\phi=0, \pm \frac{1}{2} \pi, \pi$. However, there is a unique prescription for treating both relations together such that the packet produced is the rotated version (19) of (16). For any given packet, define the quantities

$$
\begin{aligned}
\mu^{2} & =\left[\Delta \cos \left(\phi-\phi_{0}\right)\right]^{2}(\Delta L)^{2} \\
& -\frac{1}{4}\left|\left\langle\sin \left(\phi-\phi_{0}\right)\right\rangle\right|^{2}, \\
\nu^{2} & =\left[\Delta \sin \left(\phi-\phi_{0}\right)\right]^{2}(\Delta L)^{2} \\
& -\frac{1}{4}\left|\left\langle\cos \left(\phi-\phi_{0}\right)\right\rangle\right|^{2} .
\end{aligned}
$$

Requiring that the quadratic combination $Q=\mu^{2}+\nu^{2}$ be the constant $Q_{0}$ given by the case $\phi_{0}=0$ yields the solution (19). The value of $Q_{0}$ is

$$
Q_{0}=\frac{\delta^{2}}{4}\left(\frac{I_{1}(2 \delta)}{I_{0}(2 \delta)}\right)^{2}\left[\frac{1}{\delta^{2}}-\left(\frac{I_{1}(2 \delta)}{I_{0}(2 \delta)}\right)^{2}+\frac{I_{2}(2 \delta)}{I_{0}(2 \delta)}\right] .
$$

\section{Features of CSS}

The form (16) of the CSS can be used to obtain second-order expectations. We find

$$
\begin{gathered}
\left\langle\cos ^{2} \phi\right\rangle=\frac{I_{1}^{\prime}(2 \delta)}{I_{0}(2 \delta)}, \\
\left\langle\sin ^{2} \phi\right\rangle=\frac{1}{2 \delta} \frac{I_{1}(2 \delta)}{I_{0}(2 \delta)}=\frac{1}{2 \delta}\langle\cos \phi\rangle,
\end{gathered}
$$

and

$$
\left\langle L^{2}\right\rangle=\frac{\delta}{2} \frac{I_{1}(2 \delta)}{I_{0}(2 \delta)}+\beta^{2}=\frac{\delta}{2}\langle\cos \phi\rangle+\beta^{2} .
$$

Note that $\left\langle\sin ^{2} \phi\right\rangle+\left\langle\cos ^{2} \phi\right\rangle=1$, as required. In the limit $\delta \rightarrow 0,\left\langle\sin ^{2} \phi\right\rangle$ and $\left\langle\cos ^{2} \phi\right\rangle$ both approach $\frac{1}{2}$, while as $\delta \rightarrow \infty,\left\langle\sin ^{2} \phi\right\rangle \rightarrow 0$ and $\left\langle\cos ^{2} \phi\right\rangle \rightarrow 1$.

The uncertainties in the angular coordinates and momentum are

$$
\begin{aligned}
(\Delta \cos \phi)^{2} & =\frac{I_{0}(2 \delta) I_{1}^{\prime}(2 \delta)-\left[I_{1}(2 \delta)\right]^{2}}{\left[I_{0}(2 \delta)\right]^{2}}, \\
(\Delta \sin \phi)^{2} & =\frac{1}{2 \delta} \frac{I_{1}(2 \delta)}{I_{0}(2 \delta)}=\frac{1}{2 \delta}\langle\cos \phi\rangle
\end{aligned}
$$




$$
(\Delta L)^{2}=\frac{\delta}{2} \frac{I_{1}(2 \delta)}{I_{0}(2 \delta)}=\frac{\delta}{2}\langle\cos \phi\rangle
$$

These confirm that the uncertainty product (11) is minimized.

The uncertainty relations reveal that the CSS parameter $\delta$ represents the angularmomentum spread of the solution $\chi(\phi)$. As $\delta$ decreases so does $\Delta L$, and hence the angular-coordinate width increases. In the limit $\delta \rightarrow 0$, the normalization constant $N_{2} \rightarrow 1 / \sqrt{2 \pi}$, so $\chi(\phi)$ tends to an angular-momentum eigenstate $Y_{\beta}(\phi)=$ $(2 \pi)^{-1 / 2} \exp (i \beta \phi)$. This state has $\Delta L \rightarrow 0$, consistent with the limit $\delta \rightarrow 0$ of Eq. (27). Moreover, $\langle\sin \phi\rangle \rightarrow 0$ also, so both sides of the uncertainty relation (10) vanish in this limit. This avoids the appearance of infinite uncertainty in $\Delta \cos \phi$, which is impossible for a bounded function. Similarly, as $\delta \rightarrow 0$ both sides of Eq. (11) vanish.

The interpretation of $\delta$ as the angular-momentum spread is confirmed by an expansion of the packet (16) in the vicinity of $\phi=0:|\chi(\phi)|^{2} \propto \exp \left[2 \delta\left(1-\frac{1}{2} \phi^{2}+\cdots\right)\right]$. This shows that to leading order $|\chi(\phi)|^{2}$ has a gaussian dependence proportional to $\exp \left(-\delta \phi^{2}\right)$, with angular-coordinate standard deviation $\sigma=1 / \sqrt{2 \delta}$. It also implies that a CSS of finite angular-coordinate width satisfies $|\langle\cos \phi\rangle|<1$ along with the conditions (13), which differs from the corresponding classical particle located on the unit circle at $\cos \phi=1, \sin \phi=0$.

Given the initial angular location, a CSS is specified by two quantities $\delta$ and $\beta$. Compared to the corresponding classical problem, an extra parameter is needed to fix the quantum solution. Classically, the motion on a circle is determined by the initial values of $\sin \phi$, the $\operatorname{sign}$ of $\cos \phi$, and $L$. At the quantum level, the initial angular position is specified by $\langle\sin \phi\rangle$ and the $\operatorname{sign}$ of $\langle\cos \phi\rangle$, while $\beta=\langle L\rangle$ establishes the angular momentum. The extra parameter $\delta$ controls the angular spread of the packet and can be fixed through Eq. (24) by giving $\Delta L$. Evidently, in an experiment attempting to produce a wave packet localized in the angle $\phi$, it is insufficient to excite a state of definite $l$. A single laser pulse alone therefore cannot excite an angular wave packet. A superposition of $l$ states with a spread $\Delta L$ must be produced by turning on additional fields that excite more than one value of $l$.

Plots of some normalized CSS as a function of $\phi$ are presented in Fig. 1. The configurations all have $\langle\sin \phi\rangle=0,\langle\cos \phi\rangle>0$, and $\beta=\langle L\rangle=30$. Three different 
examples are shown, with $\Delta L=0.5,1.5$, and 2.5 corresponding to $\delta \simeq 0.804,4.757$, and 12.753, respectively. As expected, increasing $\delta$ decreases the angular-coordinate spread.

\section{ELLIPTICAL SQUEEZED STATES}

This section discusses the elliptical squeezed states. Sec. IVA constructs them and explains how a given ESS is specified in terms of physical quantities. The issue of time evolution is examined in Sec. IVB, where we show that the ESS move along an orbit close to a classical ellipse. In Sec. IVC, features of the ESS solutions and their relationship to other approaches are considered.

\section{A. Construction and Specification of ESS}

The CSS solution (16) is a function only of the angular coordinates and has neither time dependence nor dependence on the energy quantum number $n$. One solution of the full planar Coulomb problem is the the product of a CSS with a radial energy eigenstate of given $n$. However, this produces a stationary state.

The desired closest-to-classical packet moving on a keplerian orbit can be obtained by taking advantage of the separability of the full hamiltonian. Note that the uncertainty relation (田) is independent of $l$ because the uncertainty $\Delta R$ involves the combination $R-\langle R\rangle$. This implies it is possible to minimize simultaneously the uncertainty relations (4) and (10) at a given time. We can therefore take as an initial state the product of an $\operatorname{RSS} \psi(r)$ and a CSS $\chi(\phi)$, giving

$$
\begin{aligned}
\Psi(r, \phi) & =\psi(r) \chi(\phi) \\
& =\left(\frac{\left(2 \gamma_{0}\right)^{2 \alpha+2}}{2 \pi I_{0}(2 \delta) \Gamma(2 \alpha+2)}\right)^{\frac{1}{2}} r^{\alpha} \exp \left[-\left(\gamma_{0}+i \gamma_{1}\right) r+\delta \cos \phi+i \beta \phi\right],
\end{aligned}
$$

where we have substituted from Eqs. (5) and (16). This is a normalized five-parameter family of ESS. They represent minimum-uncertainty packets localized in both radial and angular coordinates and evolving in time. As discussed in Sec. III, the choice of the initial angular-coordinate location is implicit in the CSS construction and is taken to be $\langle\sin \phi\rangle=0$ and $\langle\cos \phi\rangle>0$. 
Expectation values of operators in the ESS can be calculated analytically using Eq. (28). Some physically useful expectations are as follows:

$$
\begin{gathered}
\langle r\rangle=\frac{\alpha+1}{\gamma_{0}}, \quad\left\langle r^{2}\right\rangle=\frac{(\alpha+1)(2 \alpha+3)}{2 \gamma_{0}^{2}}, \\
\left\langle p_{r}\right\rangle=-\gamma_{1} \quad, \quad\left\langle p_{r}^{2}\right\rangle=\frac{\gamma_{0}^{2}}{2 \alpha}+\gamma_{1}^{2}, \\
\langle\sin \phi\rangle=0 \quad, \quad\langle\cos \phi\rangle=\frac{I_{1}(2 \delta)}{I_{0}(2 \delta)}>0, \\
\langle L\rangle=\beta, \quad\left\langle L^{2}\right\rangle=\frac{\delta}{2} \frac{I_{1}(2 \delta)}{I_{0}(2 \delta)}+\beta^{2}, \\
\langle H\rangle=\frac{\gamma_{0}\left(\gamma_{0}-4\right)}{2(2 \alpha+1)}+\frac{\gamma_{1}^{2}}{2}+\frac{\gamma_{0}^{2}}{\alpha(2 \alpha+1)}\left(\frac{\delta}{2} \frac{I_{1}(2 \delta)}{I_{0}(2 \delta)}+\beta^{2}\right)
\end{gathered}
$$

The expectation value for the energy $\langle H\rangle$ is obtained using the planar Coulomb hamiltonian and depends on all five of the parameters associated with the component RSS and CSS.

The associated uncertainty products are given by

$$
\begin{aligned}
\Delta r \Delta p_{r} & =\frac{1}{2} \sqrt{\frac{\alpha+1}{\alpha}} \\
\Delta \sin \theta \Delta L & =\frac{1}{2} \frac{I_{1}(2 \delta)}{I_{0}(2 \delta)} \\
\Delta \cos \theta \Delta L & =\frac{1}{2} \frac{I_{0}(2 \delta) I_{1}(2 \delta) I_{1}^{\prime}(2 \delta)-\left[I_{1}(2 \delta)\right]^{3}}{\left[I_{0}(2 \delta)\right]^{3}}
\end{aligned}
$$

The ESS is not a minimum-uncertainty state in $r$ and $p_{r}$, which is as expected since it is constructed to minimize the uncertainty relation (đ) instead. For large values of $\alpha$, however, $\Delta r \Delta p_{r} \rightarrow \frac{1}{2}$.

We consider an initial configuration where the wave packet is located at the outer apsidal point of an elliptical orbit. This choice is consistent with the experimental situation, where the uncertainty product for radial Rydberg packets reaches a minimum near the outer apsidal point [21. The constraints imposed on $\langle\sin \phi\rangle$ and $\langle\cos \phi\rangle$ ensure that the ellipse has semimajor axis aligned along the $x$ axis of the coordinate system. The parameters $\alpha, \beta, \gamma_{0}, \gamma_{1}$ and $\delta$ can be fixed in terms of the spread $\Delta L$ 
in the angular momentum and expectations of the radial coordinates $\langle r\rangle$ and $\left\langle p_{r}\right\rangle$, of the angular momentum $\langle L\rangle$, and of the energy $\langle H\rangle$.

Classically, $\langle r\rangle,\left\langle p_{r}\right\rangle$, and $\langle L\rangle$ determine the mean location and initial velocity of the packet. At the quantum level, two additional physical conditions must be given to determine completely the ESS. These conditions represent the initial width of the packet in the radial and angular coordinates. For the first, we set the energy expectation equal to the mean energy of a Rydberg packet consisting of a superposition of $n$ states centered on the value $\bar{n}$. A packet of this type can be produced by excitation with a short laser pulse tuned to the mean energy $E_{\bar{n}} \equiv-1 / 2(\bar{n}-1 / 2)^{2}$. If the excitation occurs in the presence of external fields, a superposition of $l$ states can be achieved as well. The second extra condition is the spread $\Delta L$ in this superposition. The precise field arrangement will determine $\Delta L$, although in practice it may be difficult to determine a priori for a given experiment.

Denote by $\bar{l}$ the average value of $l$ for the superposition of $l$ states. Then, we choose to set the expectation of the coordinate $r$ equal to a radial distance to the outer apsidal point written in terms of these average quantum numbers,

$$
r_{\text {out }}=\left(\bar{n}-\frac{1}{2}\right)^{2}\left(1+\sqrt{1-\frac{\bar{l}^{2}}{\left(\bar{n}-\frac{1}{2}\right)^{2}}}\right) \text {. }
$$

The expectation of the radial momentum is set to zero. These choices are not uniquely enforced by experimental considerations but do ensure that both RSS and CSS are readily recovered in suitable limits of the ESS.

The full set of conditions sufficient to fix the five ESS parameters is therefore as follows:

$$
\begin{gathered}
\langle r\rangle=r_{\text {out }}, \quad\left\langle p_{r}\right\rangle=0 \quad, \quad\langle L\rangle=\bar{l}, \\
\langle H\rangle=E_{\bar{n}}, \quad \sqrt{\left\langle L^{2}\right\rangle-\langle L\rangle^{2}}=\Delta L
\end{gathered}
$$

These determine $\alpha, \beta, \gamma_{0}, \gamma_{1}$, and $\delta$ in terms of the three quantities $\bar{n}, \bar{l}$, and $\Delta L$ whose values depend on a particular excitation scheme using a short-pulsed laser in 
the presence of external fields.

\section{B. Evolution of ESS}

With parameters matching a Rydberg wave packet at the outer apsidal point on an elliptical orbit, the ESS (28) may be taken as a minimum-uncertainty initial solution of the time-dependent Schrödinger equation. Since $\left\langle p_{r}\right\rangle=0$ and $\langle L\rangle=\beta$ by construction, for $\beta>0$ the wave packet will begin to move in the direction of increasing $\phi$. The geometry of the ensuing orbit depends on the values of $\bar{n}, \bar{l}$, and $\Delta L$. In the limit $\bar{l} \rightarrow n-1$, the orbits for the ESS should become more circular in shape, and the wave packet should propagate at a fixed mean radial distance from the nucleus. As $\bar{l} \rightarrow 1$, the orbit should become highly elliptical, and the inner apsidal point should approach the origin. In this case, radial oscillations should occur as the particle passes close to the nucleus, as is observed for $l=1$ RSS [12].

The time evolution of the ESS may be studied by expanding $\Psi(r, \phi, t)$ in eigenstates $R_{n l}(r)$ of the energy $E_{n}=-1 / 2(n-1 / 2)^{2}$ and $Y_{l}(\phi)$ of the angular momentum, given by

$$
R_{n l}(r)=N r^{|l|} e^{-\frac{r}{n-1 / 2}} L_{n-|l|-1}^{2|l|}\left(\frac{2 r}{n-\frac{1}{2}}\right) \quad, \quad Y_{l}(\phi)=\frac{1}{\sqrt{2 \pi}} \exp (-i l \phi),
$$

where $N$ is a normalization constant, $n$ is a positive integer, and $l$ is a positive or negative integer satisfying $|l| \leq(n-1)$. The expansion of the ESS is

$$
\Psi(r, \phi, t)=\sum_{n, l} c_{n l} R_{n l}(r) Y_{l}(\phi) e^{-i E_{n} t}
$$

Note that the time dependence of the phase means that the ESS is separable only at $t=0$.

The expansion coefficients $c_{n l}=\left\langle\Psi(r, \phi, 0) \mid R_{n l}(r) Y_{l}(\phi)\right\rangle$ may be calculated using Eq. (28) as the initial wave function $\Psi(r, \phi, 0)$. The result for $l \geq 0$ is

$$
\begin{gathered}
c_{n l}=\left[\frac{\left(2 \gamma_{0}\right)^{2 \alpha+2}}{I_{0}(2 \delta) \Gamma(2 \alpha+2)}\right]^{\frac{1}{2}}\left[\frac{1}{(2 n-1)}\left(\frac{2}{n-\frac{1}{2}}\right)^{2 l+2} \frac{\Gamma(n-l)}{\Gamma(n+l)}\right]^{\frac{1}{2}} I_{\beta-l}(\delta) \\
\times \sum_{p=0}^{n-l-1}\left(\frac{-2}{n-\frac{1}{2}}\right)^{p} \frac{\Gamma(n+l) \Gamma(\alpha+l+p+2)}{p ! \Gamma(p+2 l+1) \Gamma(n-l-p)}\left(\frac{1}{n-\frac{1}{2}}+\gamma_{0}+i \gamma_{1}\right)^{-(\alpha+l+p+2)} .
\end{gathered}
$$


As an example, consider a wave packet with $\bar{n}=45, \bar{l}=30$, and $\Delta L=2.5$. Using these values and Eq. (38), we obtain the ESS-parameter values $\alpha \simeq 57.408, \beta=30$, $\gamma_{0} \simeq 0.01697, \gamma_{1}=0$, and $\delta \simeq 12.753$. The series in (40) may be well approximated by truncating the sum to a finite number of terms centered on $\bar{n}$ and $\bar{l}$. This permits the ESS to be plotted as a function of $r$ and $\phi$ at different times $t$.

Figure $2 \mathrm{a}$ shows the initial ESS defined at $t=0$. It is located on the positive $x$ axis at the outer apsidal point and is evidently localized in both radial and angular coordinates. The radial distance to the outer apsidal point is $r_{\text {out }} \simeq 3443$ in atomic units.

The classical orbital period for motion on a keplerian ellipse is $T_{\mathrm{cl}}=2 \pi(\bar{n}-1 / 2)^{3}$. With $\bar{n}=45$, we obtain $T_{\mathrm{cl}} \simeq 13.4$ psec. Figure $2 \mathrm{~b}$ shows the ESS at the time $t=\frac{1}{3} T_{\mathrm{cl}}$. It has moved in the direction of positive $\phi$ and is spreading along the elliptical orbit. Since the packet moves slowly near the outer apsidal point but faster near the inner apsidal point, in accordance with Kepler's laws, it has traversed less than one third of the orbit at $t=\frac{1}{3} T_{\mathrm{cl}}$.

Figure 2c shows the ESS at $t=\frac{1}{2} T_{\mathrm{cl}}$. It is moving rapidly and has substantial spread along the elliptical orbit. Since $\bar{l}=30$ is relatively large for a state with $\bar{n}=45$, the ESS remains at some distance from the nucleus and therefore exhibits none of the radial oscillations seen for RSS p states [12]. The radial distance to the inner apsidal point is $r_{\text {in }} \simeq 517$ a.u.

Figures $2 \mathrm{~d}$ and $2 \mathrm{e}$ show the wave packet at the times $t=\frac{2}{3} T_{\mathrm{cl}}$ and $t=T_{\mathrm{cl}}$, respectively. The motion is slower again as the outer apsidal point is approached, and the packet becomes more localized again. At $t=T_{\mathrm{cl}}$, the wave packet closely resembles the initial wave packet. However, the motion is not exactly periodic. For times $t \gg T_{\mathrm{cl}}$, the wave packet collapses and a cycle of revivals and superrevivals commences.

The localization in the angle $\phi$ oscillates as the wave packet goes through a classical orbital cycle. At $t=0$, it is highly localized in angle, while at $t=\frac{1}{2} T_{\mathrm{cl}}$, the wave packet is extended around much of the orbit. By $t=T_{\mathrm{cl}}$, the wave packet has localized again. This oscillation is characteristic of a squeezed state. The radial uncertainties are oscillating also, although not as noticeably as for a p-state radial wave function. 
In Fig. 3, two ESS with different values of $\bar{l}$ and hence different orbital eccentricities are compared. Both packets have $\bar{n}=45$ and $\Delta L=2.5$. They are viewed at $t=\frac{1}{2} T_{\mathrm{cl}}$ from a point on the positive $x$ axis looking towards the nucleus. Figure 3a, with $\bar{l}=30$, is the same as Fig. $2 \mathrm{c}$ but viewed from a different perspective. The ellipticity of the orbit is manifest. Figure $3 \mathrm{~b}$ shows a different ESS with $\bar{l}=40$, for which $\alpha \simeq 20.412, \beta=40, \gamma_{0} \simeq 0.00752, \gamma_{1}=0$, and $\delta \simeq 12.753$. Since $\Delta L$ is unchanged, $\delta$ remains the same. As expected, the orbit is closer to circular. For this case, $r_{\text {in }} \simeq 1113$ a.u., which is more than twice the value for the $\bar{l}=30$ wave packet in Fig. 3a.

\section{Features of ESS}

The literature contains examples of quantum wave packets other than the ESS that are nonetheless localized on classical elliptical orbits. In this subsection, we discuss a few additional features of the ESS and provide a comparison with some of these other approaches [8, 9].

The methods of refs. [8, 9] both involve the Runge-Lenz vector. In the classical Coulomb problem, this vector is a conserved quantity with magnitude equal to the eccentricity of the orbit and with orientation along the semimajor axis pointing from the focus to the inner apsidal point. At the quantum level, the Runge-Lenz operator in atomic units takes the form

$$
\vec{A}=\frac{1}{2}(\vec{p} \times \vec{L}-\vec{L} \times \vec{p})-\frac{\vec{r}}{r}
$$

In ref. [8], the Runge-Lenz operator is scaled by a factor involving the energy operator at a fixed value of $n$. Within a fixed $n$ manifold, the Coulomb problem exhibits an $\mathrm{SO}(4)$ symmetry. Coherent states for the $\mathrm{SO}(4)$ symmetry can be constructed using the scaled Runge-Lenz operator and the angular-momentum operator. The resulting wave packets are localized on elliptical orbits. However, they are stationary states that do not follow the classical motion.

A similar approach is taken in ref. [9]. Planar motion is considered, and a subset of the operators is used to form an o(3) algebra. The operators $A_{x}, A_{y}$, and $L$ satisfy

$$
\left[A_{x}, A_{y}\right]=-2 i H L
$$


where $H$ is the hamiltonian. The associated uncertainty relation is

$$
\Delta A_{x} \Delta A_{y} \geq|\langle H L\rangle|
$$

Quantum solutions minimizing this relation are found. The resulting states are also stationary and are elliptical in shape. By taking a gaussian-weighted superposition of these states with different values of $n$, a localized wave packet is produced that moves along a keplerian orbit and follows the classical motion. In the limit of large angular quantum numbers, Eq. (44) approximately holds for these superpositions.

The ESS are different from the solutions of refs. [8, 9]. They naturally contain a superposition of $n$ and $l$ states, and they are minimum-uncertainty solutions for both radial and angular operators. The shape of the elliptical orbit is determined by the parameters of the ESS, which in turn depend on the experimentally determined parameters $\bar{n}, \bar{l}$, and $\Delta L$.

A natural question to ask is whether the ESS also provide a minimum-uncertainty solution of Eq. (44). For the example plotted in Fig. 2, the values of the ESS parameters given in Sec. IV can be used to determine numerically the left and right hand sides of (44). We find $\Delta A_{x} \Delta A_{y} \simeq 0.1214$, while $|\langle H L\rangle| \simeq 0.0099$ in atomic units. Defining the quantity

$$
Z=\frac{\Delta A_{x} \Delta A_{y}-|\langle H L\rangle|}{|\langle H L\rangle|},
$$

we find $Z \simeq 11.26$ for the ESS wave function in Fig. 2a. A minimum-uncertainty solution of Eq. (44) would have $Z=0$.

Another interesting issue is the relationship between the ESS parameters $\alpha, \beta$, $\gamma_{0}, \gamma_{1}, \delta$ and quantities determining the shape of the corresponding classical orbital ellipse, namely, the semimajor axis $a$, the angle $\eta$ it makes with the $x$ axis, and the eccentricity $e$. The Ehrenfest theorem requires that the mean values of the position $\langle\vec{r}\rangle$ and the momentum $\langle\vec{p}\rangle$ evolve according to the equations $d\langle\vec{r}\rangle / d t=\langle\vec{p}\rangle$ and $d\langle\vec{p}\rangle / d t=-\langle\vec{\nabla} V(\vec{r})\rangle$. This means that the mean position moves along the classical trajectory if $\langle\vec{\nabla} V(\vec{r})\rangle=\vec{\nabla} V(\langle\vec{r}\rangle)$. However, this condition is not satisfied for the Coulomb problem. The connection between the ESS parameters and the shape of the corresponding classical orbit is therefore difficult to establish analytically and is partly a matter of definition. 
If the quantum motion did follow the classical trajectory, knowledge of the initial position and momentum would suffice to determine it. One useful approximate relationship between the ESS parameters and those of the classical orbit in cartesian coordinates is therefore given by taking ESS expectations of suitable quantities as the initial data for a classical trajectory and thereby establishing the desired relation. This procedure has the advantage of being analytical and providing insight about the meaning of the ESS parameters.

For present purposes, we choose to express the ESS parameters as functions of $a$, $\eta, e$, and the widths $\Delta r$ and $\delta$ of the ESS, which have no direct classical counterpart. We find

$$
\begin{aligned}
\gamma_{0} & =\frac{I_{0}(2 \delta)}{I_{1}(2 \delta)} \frac{a}{2(\Delta r)^{2}}\left(1+e \cos \eta-\frac{e^{2} \sin ^{2} \eta}{1-e \cos \eta}\right) \\
\alpha & =2 \gamma_{0}^{2}(\Delta r)^{2}-1 \\
\beta^{2} & =\frac{(2 \alpha+1)^{2}}{4 \gamma_{0}(\alpha+1)}(1-e \cos \eta)\left(\frac{I_{0}(2 \delta)}{I_{1}(2 \delta)}\right)^{3}, \\
\gamma_{1} & =-\frac{2 \alpha+1}{2 \beta(\alpha+1)} e \sin \eta\left(\frac{I_{0}(2 \delta)}{I_{1}(2 \delta)}\right)^{3} .
\end{aligned}
$$

Note that there are two solutions to the equation for $\beta$, reflecting the two possible directions the trajectory is followed. For the examples considered above, $\eta=0$ by assumption, corresponding to an ESS located at the outer apsidal point.

As an example of the use of these expressions, we can address the general issue of whether Eq. (44) is minimized by an ESS for any shape of classical orbit. For definiteness, choose $\Delta r$ and $\Delta L$ to match the example in Fig. 2, and take $\eta=0$. Then, the expressions (49) can be used to obtain the quantity $Z$ of Eq. (45) as a function of the semimajor axis $a$ and eccentricity $e$. Figure 4 shows a plot of the resulting lengthy expression for $Z$, which provides a normalized measure of closeness to minimum uncertainty in Eq. (44). The value $Z=0$ corresponds to minimum uncertainty. Figure 4 shows that $Z>0$ for the full range of associated classical orbits displayed in the graph. This demonstrates more generally that the ESS are different 
from the superposition of $\mathrm{O}(3)$ coherent states presented in ref. [9].

\section{INCORPORATION OF QUANTUM DEFECTS}

Most experiments studying the properties of Rydberg wave packets are performed using alkali-metal atoms. These have quantum defects causing energy-level shifts away from the hydrogenic values. It is therefore useful to have a relatively simple and analytical theory for alkali-metal atoms that has eigenstates with the attractive properties of hydrogenic eigenfunctions such as completeness and orthogonality, but that generates exact quantum-defect energies. A model satisfying these criteria, called supersymmetry-based quantum-defect theory (SQDT), is known to exist [32]. In this section, we outline the planar analog of SQDT and sketch the construction of the associated ESS. These may be of use in modeling packets produced in alkali-metal atoms.

The physical quantum defects $\delta(n, l)$ are empirical parameters depending in general on $n$ and $l$. We define the planar analogues $\delta(n,|l|)$ as functions of the absolute value $|l|$, so that a planar Rydberg series can be established having features in common with the standard case. As usual, the quantum defects for large $n$ approach asymptotic values $\delta(|l|)$ that are independent of $n$.

The planar Rydberg series has the form

$$
E_{n^{*}}=\frac{-1}{2\left(n^{*}-\frac{1}{2}\right)^{2}},
$$

where $n^{*}=n-\delta(|l|)$. The quantum defects partially lift the degeneracy among states having different values of $|l|$. The primary objective of planar SQDT is to obtain analytical eigenfunctions with (50) as eigenenergies. This is accomplished by defining an effective radial potential

$$
V(r)=-\frac{1}{r}+\frac{\left|l^{*}\right|^{2}-|l|^{2}}{2 r^{2}},
$$

where $\left|l^{*}\right|=|l|-\delta(|l|)+I(|l|)$ and $I(|l|)$ is an integer that depends on $|l|$. The radial eigenstates $R_{n^{*} l^{*}}(r)$ of this potential have the form of $R_{n l}$ in Eq. (39) but with $n$ and $|l|$ replaced by $n^{*}$ and $\left|l^{*}\right|$, respectively. For certain values of the integers $I(|l|)$ and 
in the limit of vanishing quantum defects, these solutions admit a supersymmetric extension.

The set of planar SQDT eigenstates $R_{n^{*} l^{*}}(r) Y_{l}(\phi)$, where $Y_{l}$ is an eigenstate of angular momentum, is complete and orthonormal. We may choose $\delta(|l|)$ to match the asymptotic quantum defects $\delta(l)$, with $l \geq 0$, for the physical alkali-metal atom. For example, for lithium s and p states, $\delta(0) \simeq 0.40$ and $\delta(1) \simeq 0.05$. With these values, the planar SQDT eigenstates reproduce the planar Rydberg series for lithium.

An interesting issue is the ESS construction in the context of this model. At the classical level, the presence of the modified $1 / r^{2}$ term in the effective potential (51) means the Runge-Lenz vector $\vec{A}$ is no longer conserved. The orbit precesses at a rate determined by the quantum defect and is no longer a keplerian ellipse. At the quantum level, the commutator of the Runge-Lenz vector with the hamiltonian is nonzero, and the $\mathrm{SO}(4)$ symmetry is broken.

Despite these differences, the RSS construction can be performed in the presence of quantum defects [13]. For the present case, the oscillator variables $R$ and $P$ must be modified to

$$
R=\frac{1}{r}-\frac{1}{\left|l^{*}\right|^{2}} \quad, \quad P=-\frac{i}{f}\left(\partial_{r}+\frac{1}{2 r}\right),
$$

where $f=\left|l^{*}\right| /|l|$. These operators obey the uncertainty relation

$$
\Delta R \Delta P \geq \frac{1}{2 f}\left\langle\frac{1}{r^{2}}\right\rangle
$$

which also depends on the quantum defect. The wave function $\psi(r)$ minimizing this relation has the same functional form as that in Eq. (5).

An $\operatorname{ESS} \Psi(r, \phi)$ can be formed as a product of an $\operatorname{RSS} \psi(r)$ and a CSS $\chi(\phi)$. The form of the solution is like that in Eq. (28). However, some of the parameters must be chosen differently. We choose $\left\langle p_{r}\right\rangle=0$ and $\langle L\rangle=\bar{l}$, and we specify $\Delta L$ as before. However, $\langle H\rangle=E_{\bar{n}^{*}}$ and $\langle r\rangle=r_{\text {out }}^{*}$ differ from the previous case. Here, $E_{\bar{n}^{*}}$ is the energy expectation and $r_{\text {out }}^{*}$ is the outer apsidal point for a superposition of states with quantum-defect eigenenergies. This means the ESS is initially located at the outer apsidal point of a precessing ellipse. The time evolution and revival structure depend on the quantum defects and can be studied by expanding $\Psi(r, \phi, t)$ in the complete set of SQDT eigenstates. 
In the above, a prescription for calculating $\langle H\rangle$ is needed since the SQDT potential depends on $|l|$ and $\left|l^{*}\right|$. We can take advantage of completeness to expand the initial ESS as a superposition of SQDT eigenstates:

$$
\Psi(r, \phi)=\sum_{n, l} \tilde{c}_{n l} R_{n^{*} l^{*}}(r) Y_{l}(\phi)
$$

where the expansion coefficients $\tilde{c}_{n l}$, which depend on the ESS parameters, can be

determined by inversion. The expectation value for the hamiltonian can then be specified as

$$
\langle H\rangle=\sum_{n, l}\left|\tilde{c}_{n l}\right|^{2} E_{n^{*}}=E_{\bar{n}^{*}}
$$

\section{SUMMARY}

In this paper, we have found analytical solutions to the planar Coulomb problem, called elliptical squeezed states, that minimize coordinate-momentum uncertainty relations and that move along classical keplerian orbits. The paper also provides an extension of the analysis to the case where quantum defects are present, which is of experimental importance but theoretically difficult to treat in other approaches because quantum defects break the $\mathrm{O}(4)$ symmetry.

The ESS provide an analytical tool for studying the quantum-classical correspondence in the Coulomb problem. They may also be used to describe minimumuncertainty Rydberg wave packets excited by short laser pulses with external fields present. To match an ESS to a Rydberg wave packet, we initialize the ESS at the outer apsidal point of the orbit. The five ESS parameters are given in terms of the expectation values of the radial position, the radial momentum, the energy, the angular momentum, and the spread in the angular momentum.

We have studied the time evolution of the ESS for examples with different semimajor axes and eccentricities. The wave packets move along an elliptical trajectory with the classical keplerian orbital period. The squeezing of the ESS is evident as the width of the wave packet oscillates during the motion. The ESS maintain their form for several orbital cycles before decoherence. 


\section{REFERENCES}

1. See, for example, J.R. Klauder and B.-S. Skagerstam, eds., Coherent States (World Scientific, Singapore, 1985); D.H. Feng, J.R. Klauder, and M.R. Strayer, eds., Coherent States (World Scientific, Singapore, 1994).

2. E. Schrödinger, Naturwissenschaften 14, 664 (1926).

3. Some correspondence concerning his efforts can be found in E. Schrödinger, Collected Papers on Wave Mechanics (Blackie and Son, London, 1958).

4. L.S. Brown, Am. J. Phys. 41, 525 (1973).

5. J. Mostowski, Lett. Math. Phys. 2, 1 (1977).

6. M.M. Nieto, Phys. Rev. D 22, 391 (1980); V.P. Gutschick and M.M. Nieto, Phys. Rev. D 22, 403 (1980).

7. D.S. McAnally and A.J. Bracken, J. Phys. A 23, 2027 (1990).

8. J.-C. Gay, D. Delande, and A. Bommier, Phys. Rev. A 39, 6587 (1989).

9. M. Nauenberg, Phys. Rev. A 40, 1133 (1989).

10. I. Zlatev, W.-M. Zhang, and D.H. Feng, Phys. Rev. A 50, R1973 (1994).

11. For discussions of the nature and role of squeezed states, see, for example, in D. Han, Y.S. Kim, and W.W. Zachary, eds., Proceedings of the Third International Workshop on Squeezed States and Uncertainty Relations, NASA, Washington, D.C., 1994.

12. R. Bluhm and V.A. Kostelecký, Phys. Rev. A 48, R4047 (1993) (quantph/9508019).

13. R. Bluhm and V.A. Kostelecký, Phys. Rev. A 49, 4628 (1994) (quantph/9508020).

14. M.M. Nieto, in G.T. Moore and M.O. Scully, eds., Frontiers of Nonequilibrium Statistical Physics (Plenum, New York, 1986).

15. A. ten Wolde, L.D. Noordam, A. Lagendijk, and H.B. van Linden van den Heuvell, Phys. Rev. Lett. 61, 2099 (1988).

16. J.A. Yeazell, M. Mallalieu, J. Parker, and C.R. Stroud, Phys. Rev. A 40, 5040 
(1989).

17. J.A. Yeazell, M. Mallalieu, and C.R. Stroud, Phys. Rev. Lett. 64, 2007 (1990).

18. J.A. Yeazell and C.R. Stroud, Phys. Rev. A 43, 5153 (1991).

19. D.R. Meacher, P.E. Meyler, I.G. Hughes, and P. Ewart, J. Phys. B 24, L63 (1991).

20. J. Wals, H.H. Fielding, J.F. Christian, L.C. Snoek, W.J. van der Zande, and H.B. van Linden van den Heuvell, Phys. Rev. Lett. 72, 3783 (1994).

21. J. Parker and C.R. Stroud, Phys. Rev. Lett. 56, 716 (1986); Phys. Scr. T12, 70 (1986).

22. G. Alber, H. Ritsch, and P. Zoller, Phys. Rev. A 34, 1058 (1986); G. Alber and P. Zoller, Phys. Rep. 199, 231 (1991).

23. I.Sh. Averbukh and N.F. Perelman, Phys. Lett. 139A, 449 (1989).

24. M. Nauenberg, J. Phys. B 23, L385 (1990).

25. R. Bluhm and V.A. Kostelecký, Phys. Rev. A 50, R4445 (1994) (hepph/9410325).

26. R. Bluhm and V.A. Kostelecký, Phys. Lett. A 200, 308 (1995) quantph/9508024); Phys. Rev. A 51, 4767 (1995) (quant-ph/9506009).

27. J.A. Yeazell and C.R. Stroud, Phys. Rev. Lett. 60, 1494 (1988).

28. Z.D. Gaeta, M. Noel, and C.R. Stroud, Phys. Rev. Lett. 73, 636 (1994).

29. P. Carruthers and M.M. Nieto, Rev. Mod. Phys. 40, 411 (1968).

30. W.H. Louisell, Phys. Lett. 7, 60 (1963).

31. V.A. Kostelecký and B. Tudose, Indiana University Report No. IUHET 292 (1995).

32. V. A. Kostelecký and M. M. Nieto, Phys. Rev. A 32, 3243 (1985). For a review, see V.A. Kostelecký, in B. Gruber and T. Osaka, eds., Symmetries in Science VII: Dynamic Symmetries and Spectrum-Generating Algebras in Physics, Plenum, New York, 1993 (quant-ph/9508015). 


\section{FIGURE CAPTIONS}

Fig. 1: Sample CSS $|\chi(\phi)|^{2}$ (arbitrary units) plotted as a function of $\phi$ in radians. All three cases shown are centered on $\phi=0$ with $\beta=\langle L\rangle=30$. The angularcoordinate widths of the CSS depend on $\delta$ and have the values $\Delta L=0.5$ for $\delta \simeq 0.804$ (solid line), $\Delta L=1.5$ for $\delta \simeq 4.757$ (dotted line), and $\Delta L=2.5$ for $\delta \simeq 12.753$ (thick solid line).

Fig. 2: The modulus of an ESS $r|\Psi(r, \phi, t)|^{2}$ (arbitrary units) plotted as a function of $r$ and $\phi$ at the times: (a) $t=0$, (b) $t=\frac{1}{3} T_{\mathrm{cl}}$, (c) $t=\frac{1}{2} T_{\mathrm{cl}}$, (d) $t=\frac{2}{3} T_{\mathrm{cl}}$, (e) $t=T_{\mathrm{cl}}$.

Fig. 3: Comparison of the modulus squared (arbitrary units) of two ESS with different eccentricities at the time $t=\frac{1}{2} T_{\mathrm{cl}}$. Both wave packets have $\bar{n}=45$ and $\Delta L=$ 2.5. and are viewed from a point on the positive $x$ axis looking in toward the origin. For Figure (a), $\bar{l}=30$, while for Figure (b), $\bar{l}=40$.

Fig. 4: A plot of the quantity $Z=\left(\Delta A_{x} \Delta A_{y}-|\langle H L\rangle|\right) /|\langle H L\rangle|$ as a function of the semimajor axis $a$ in atomic units and the eccentricity $e$ of the associated classical orbits. 
This figure "fig1-1.png" is available in "png" format from: http://arxiv.org/ps/quant-ph/9509010v1 
This figure "fig2-1.png" is available in "png" format from: http://arxiv.org/ps/quant-ph/9509010v1 
This figure "fig3-1.png" is available in "png" format from: http://arxiv.org/ps/quant-ph/9509010v1 
This figure "fig1-2.png" is available in "png" format from: http://arxiv.org/ps/quant-ph/9509010v1 
This figure "fig2-2.png" is available in "png" format from: http://arxiv.org/ps/quant-ph/9509010v1 
This figure "fig3-2.png" is available in "png" format from: http://arxiv.org/ps/quant-ph/9509010v1 
This figure "fig1-3.png" is available in "png" format from: http://arxiv.org/ps/quant-ph/9509010v1 
This figure "fig2-3.png" is available in "png" format from: http://arxiv.org/ps/quant-ph/9509010v1 
This figure "fig3-3.png" is available in "png" format from: http://arxiv.org/ps/quant-ph/9509010v1 